\title{
Concurrent exercise improves insulin resistance and nonalcoholic fatty liver disease by upregulating PPAR- $\gamma$ and genes involved in the beta-oxidation of fatty acids in ApoE-KO mice fed a high-fat diet
}

\author{
Fan Zheng and Ying Cai ${ }^{*}$ (i)
}

\begin{abstract}
Objective: To emphasize the mechanism of concurrent exercise effect on lipid disorders in insulin resistance (IR) and nonalcoholic fatty liver disease (NAFLD).

Materials and methods: Twenty male ApoE knockout mice were randomly divided into two groups: HFD group $(n=10)$ fed a high fat diet, and HFDE group $(n=10)$ with high-fat diet intervention for 12 weeks and swimming exercise. Other ten healthy male C57BL/6 J mice were fed a normal diet, and included as control group. Retroorbital blood samples were collected for biochemical analysis. Oil red O staining of liver tissues was performed to confirm the exercise effect. Western blotting was performed to evaluate the expressions of PPAR- $\gamma$, CPT-1, MCAD.

Results: The levels of TG, TC, LDL, FFA, FIN, FPG and Homa-IRI in the HFD group were significantly higher than ND group, while these were markedly decreased in the HFDE group compared with HFD group. The Oil Red O staining of liver samples further confirmed the exercise effect on the change of lipid deposition in the liver. Western blotting showed increased expressions of PPAR- $\gamma$, CPT-1, MCAD induced by high fat diet were significantly downregulated by exercise.
\end{abstract}

Conclusion: A concurrent 12-week exercise protocol alleviated the lipid metabolism disorders of IR and NAFLD, probably via PPAR- $\gamma / C P T-1 / M C A D$ signaling.

Keywords: Concurrent exercise, Insulin resistance, Non-alcoholic fatty liver disease, PPAR- $\gamma$, CPT-1, MCAD

\section{Introduction}

Type 2 diabetes mellitus (T2DM) and nonalcoholic fatty liver disease (NAFLD) are pandemic metabolic diseases. They act in a "vicious circle" that accelerates worsening of the vascular complications of T2DM and aggravates NAFLD progression to liver cirrhosis and hepatocellular carcinoma [1]. In humans, T2DM is one of the most common chronic diseases with increasing incidence due to lifestyle changes. It is characterized by hyperglycemia mainly caused by impaired insulin

\footnotetext{
*Correspondence: 3386589875@qq.com

Cardiac Rehabilitation Center of Rehabilitation Department, Xiangya Hospital at Central South University, Xiangya Road 87\#, Changsha, Hunan, People's Republic of China
}

secretion and insulin resistance [2]. Insulin resistance (IR) undoubtedly plays a vital role in the pathogenesis of T2DM and other metabolic disorders, such as obesity, high blood pressure, high blood cholesterol and metabolic syndrome. Clinically, IR is strongly associated with stress, visceral adiposity, and declined cardiorespiratory fitness; also it increases the risk of cardiovascular disease. Biologically, IR is caused by elevated concentrations of free fatty acids (FFAs). Owing to excess fat intake, FFAs are deposited as triglycerides (TG) in non-fat tissues, e.g., skeletal muscle, liver, heart and pancreas [3, 4]. The impairment of fatty acid $\beta$-oxidation caused by IR leads to an abnormal accumulation of TG in the liver and the development of 
NAFLD. The most common independent risk factor for NAFLD is believed to be IR [5, 6]. Molecularly, IR is caused by an imbalance between excess nutrients or inflammatory cytokines and the cell membrane receptors $[7,8]$.

Meanwhile, NAFLD is defined as excess fat accumulation in the liver due to causes other than excess alcohol consumption [9]. It represents the most prevalent chronic liver disease in the world; nearly $70 \%$ of the overweight population has NAFLD [10]. Several studies demonstrated that NAFLD was not only closely linked to liver-related morbidity or mortality, but also to an increased risk of diabetes and cardiovascular disease [5, 11-14]. Studies further clarified that NAFLD was an independent risk factor for the development of T2DM [7]. Though NAFLD might be reversible in the early stages, it is hard to discover because of its asymptomatic nature. Along with IR, this chronic liver condition progressively worsens and is usually not recognized until the patients develop cirrhosis [15]. Therefore, it is vital to prevent and treat IR and NAFLD in the early stages.

The incidence of NAFLD is growing worldwide, because of both the rising tide of T2DM and obesity and the lack of effective treatments. Since IR is believed to be the main cause of NAFLD, drugs targeting IR may be used for the treatment of NAFLD. Some thiazolidinediones, primarily used to improve IR and treat T2DM, were proven to be effective in alleviating the hepatic steatosis and fibrosis in patients with NAFLD. However, there are few validated pharmacological interventions for NAFLD [16, 17]. The current management of NAFLD concentrates on lifestyle modification, weight loss, and exercise. Exercise has been proven to be a significant and powerful low-risk means of improving both IR [3] and NAFLD [18], but the underlying multiple mechanisms of managing the disease still remain largely unknown.

Problems with fat metabolism are associated with NAFLD. Peroxisome proliferator-activated receptors (PPAR) belong to the nuclear hormone receptor superfamily, and play a pivotal role in glucose and fatty acid metabolism. Three members of this superfamily have already been discovered: PPAR- $\alpha$, PPAR $-\beta / \delta$ and PPAR $-\gamma$ [19]. Since NAFLD is basically caused by an abnormal fat metabolism, these nuclear receptors are key modulators in the pathological course of the disease, and they are also candidate targets for treating NAFLD [20, 21]. Specifically, PPAR- $\gamma$, which is known as 'energy-balanced receptor', has been shown to be a crucial regulator in both IR [22-25] and NAFLD $[26,27]$. This is because PPAR- $\gamma$ regulates several downstream genes related to fatty acid oxidation, such as carnitine palmitoyl transferase-1 (CPT-1) [28] and medium-chain acyl-CoA dehydrogenase (MCAD) [29]. Significantly, CPT-1 initially catalyzes the transportation of fatty acids into the mitochondria for $\beta$-oxidation, followed by a further catalysis of fatty acid $\beta$-oxidation in the mitochondrial matrix by MCAD. In short, CPT-1 and MCAD are both considered to be key enzymes for lipid metabolism [25, 30, 31]. Hence, in this study, we hypothesized that exercise might ameliorate IR and NAFLD through PPAR- $\gamma /$ CPT-1/MCAD pathway regulation. To this end, we provided an experimental model of IR and NAFLD by using Apolipoprotein-E-knockout (ApoE-KO) mice, which were fed a high fat diet (HFD). ApoE is an important constituent of all plasma lipoproteins and serves as a ligand for cell-surface lipoprotein receptors such as the LDL-receptor. ApoE-KO mice spontaneously develop hypercholesterolemia and atherosclerosis when fed a standard chow. An HFD can further exacerbate these lesions and accelerate the process. Furthermore, studies established that ApoE-KO mice fed an HFD could serve as a rapid and valuable NAFLD model [32, 33]. Next, we explored the underling mechanism of the effect of exercise on IR and NAFLD by detecting PPAR- $\gamma /$ CPT-1/MCAD expressions in the liver of ApoE-KO mice fed an HFD.

\section{Materials and methods \\ Animals and experiment protocol}

Twenty male C57BL/6 J ApoE-KO mice and 10 healthy male C57BL/6 J mice were both purchased from the Beijing Vital River Laboratory Animal Technology Company (Beijing, China). All the mice were housed in microisolator cages and assayed under conditions of controlled temperature of $23-26{ }^{\circ} \mathrm{C}$ and humidity of $60 \%$ in a 12-h light/dark cycle with free access to standard chow and sterile water. After 1 week of acclimatization to the environment, twenty male C57BL/6 J ApoE-KO mice were randomly divided into two groups: High Fat Diet group (HFD group, $n=10$ ) and High Fat Diet plus Exercise group (HFDE group, $n=10$ ). These two groups were fed a high fat diet (D12451, 45\% of total calories from fat, FBSH Biotechnologhy Inc., Shanghai, China) for 12 weeks, and meanwhile, ten healthy male C57BL/6 J mice received a standard diet (D12450J, 10\% of total calories from fat, FBSH Biotechnologhy Inc.) were included in the Normal Diet group (ND group, $n=10$ ). Furthermore, HFDE group underwent swimming training (i.e., $20 \mathrm{~min}$ on day $1,30 \mathrm{~min}$ on day $2,40 \mathrm{~min}$ on day $3,50 \mathrm{~min}$ on day 4,60 min on day 5 followed by $1 \mathrm{~h}$ per day for the next 11 weeks and continued the same protocol till week 12) in a round stainless steel water tank of $70 \mathrm{dm}^{3}$ volume and $50 \mathrm{~cm}$ depth, with water temperature at $35^{\circ} \mathrm{C}$. Body weight of all the mice were monitored and recorded periodically. After 12 weeks, all the mice were sacrificed. Retro-orbital blood samples were collected for biochemical analysis. Liver tissues were immediately excised under sterile conditions followed by washing with ice-cold normal saline, and then stored at $-80^{\circ} \mathrm{C}$ for further analysis. 


\section{Biochemical analysis}

Blood samples were collected from all the mice after overnight fasting. Fasting plasma glucose (FPG) was measured from tail vein blood by Glucose meter GM300 (Rightest Inc., Taiwan, China). Fasting serum insulin level (FIN) was assayed by INS ELISA Kit (Beijing North Institute, Beijing, China). Serum total cholesterol (TC), triglyceride (TG), high-density lipoprotein cholesterol (HDL), and FFA were measured by an automatic biochemical analyzer (HITACHI Company, Japan). Friedewald equation was applied to calculate LDL, with the following equation: LDL $(\mathrm{mmol} / \mathrm{L})=(\mathrm{TC}-\mathrm{HDL}-\mathrm{TG}) / 2.2$. Homa-IRI was calculated to ascertain the establishment of $\mathrm{IR}$, with the following equation: $\mathrm{IRI}=[\mathrm{FPG}(\mathrm{mmol} / \mathrm{L}) \times$ $\mathrm{FIN}(\mu \mathrm{IU} / \mathrm{mL})] / 22.5)$.

\section{Western blot}

The liver tissues were lysed on ice using RIPA lysis buffer (Cell Signaling Technology, USA) supplemented with protease inhibitors and phosphatase inhibitors (Roche, USA), and $1 \mathrm{mM}$ PMSF (Sigma, USA). Equal amount of proteins were separated on 10\% SDS-PAGE gels. The tissues were then transferred onto nitrocellulose membranes (Bio-Rad, USA), and were subsequently blocked with $5 \%$ skimmed milk. The membranes were incubated with primary antibodies against PPAR- $\gamma$ (ab45036, Abcam, U.S.), CPT-1 (ab104662 Abcam, U.S.), MCAD (ab92461, Abcam, U.S.) and GAPDH (G9545, Sigma-Aldrich, U.S.). After washing with $0.1 \%$ Tween 20 in TBS, the membranes were incubated with horseradish peroxidase-conjugated secondary antibody (Santa Cruz Biotechnology, U.S.) and bound antibody was detected by ECL Plus Reagents (Amersham Biosciences, U.S.). Bands were quantified and analyzed by Image Pro Plus software.

\section{Histology examination}

Liver samples were excised and embedded in Tissue-Tek OCT compound for histopathological analysis. The OCT-embedded samples were serially sectioned at $4 \mu \mathrm{m}$ and stained with Oil Red $\mathrm{O}$ for the evaluation of fat deposition. To estimate adipogenesis of the liver, after removing the staining solution, the dye retained in the cells was eluted by $200 \mu \mathrm{L}$ isopropanol, and absorbance was measured at $510 \mathrm{~nm}$ by microplate reader (Bio-RAD680, Bio-Rad Inc., USA) for quantitative analysis via Image Pro Plus software (Fig. 4).

\section{Statistical analysis}

Statistical analysis was conducted using SPSS 16.0 statistical software. All the data were reported as means \pm SD. One-way analysis of variance (ANOVA) was applied to determine statistical significance among different groups and Tukey's test was used to evaluate the differences among the means of different treatments. $P<0.05$ was considered statistically significant.

\section{Results \\ Effect of exercise on body weight}

At the beginning, there was no difference in body weight among the three groups of mice. After 12 weeks, compared with the ND group, body weight was significantly higher in the HFD group $(37.25 \pm 2.43 \mathrm{~g}$ VS $27.4 \pm 0.52 \mathrm{~g}$, $p<0.05)$. Furthermore, the body weight of the HFDE group significantly decreased after 12-weeks of swimming training compared with the HFD group (30.18 \pm $1.10 \mathrm{~g}$ VS $37.25 \pm 2.43 \mathrm{~g}$; see Table $1, p<0.05$ ). The weight change trends of three groups were presented in Fig. 1.

\section{Effect of exercise on the biochemical indicators}

All the results were presented in Table 2. Results showed that the levels of TC, TG, LDL, FFA, FIN, FPG and Homa-IRI in the HFD group were significantly higher than the ND group $(p<0.05)$. However, after 12-weeks of intervention, the levels of TC, TG, LDL, FFA, FIN, FPG and Homa-IRI were markedly decreased in the HFDE group compared with the HFD group.

\section{Evaluation of liver histology}

Oil Red O staining of samples from the ND group occasionally showed scattered lipid drops without liver steatosis. Meanwhile, Oil Red O staining of samples from the HFD group showed severe fatty changes in the liver tissue and was were characterized by diffusely mixed sizes of fat bubble, but tended to have big fat bubbles. After 12 weeks of exercise intervention, the fatty proportion of the liver was significantly reduced in the HFDE group. Results showed a distinct increase in fat deposition in the liver tissue induced by an HFD, whereas swimming decreased the fat accumulation in the liver sections (Figs. 2 and 3).

\section{Expressions of PPAR- $\gamma$, CPT-1, and MCAD}

After 12 weeks of swimming exercise intervention, compared with the ND group, the protein levels of PPAR- $\gamma$, CPT- 1 and MCAD in the HFD group were significantly decreased $(p<0.01)$. Meanwhile, compared with the HFD group, the protein levels of PPAR- $\gamma$, CPT-1 and MCAD in the HFDE group were significantly increased ( $p<0.01$; Fig. 4$)$.

Table 1 The weight change of three groups during 12 weeks (Means \pm SD, $n=30$ )

\begin{tabular}{llll}
\hline Time & Weight & $\begin{array}{l}\text { Weight } \\
\text { ND group }(n=10)\end{array}$ & Weight \\
\hline Before & $20.54 \pm 0.73 \mathrm{~g}$ & $20.69 \pm 0.52 \mathrm{~g}$ & $20.83 \pm 0.48 \mathrm{~g}$ \\
After & $27.40 \pm 0.52 \mathrm{~g}$ & $37.25 \pm 1.49 \mathrm{~g}^{*}$ & $28.42 \pm 1.10 \mathrm{~g}^{\#}$
\end{tabular}

${ }^{*} p<0.05$ VS ND group, ${ }^{\#} p<0.05$ VS HFD group 


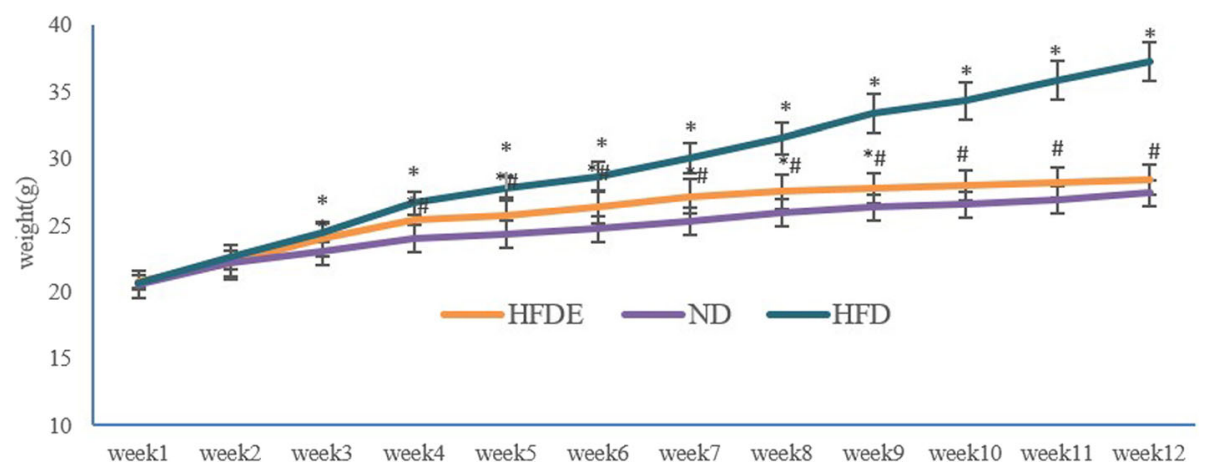

Fig. 1 The weight change of three groups (Means \pm SD). The orange line marks the HFDE group, the purple line marks the ND group, and the blue line marks the HFD group. ${ }^{*} p<0.05$ VS ND group, ${ }^{\#} p<0.05$ VS HFD group

\section{Discussion}

The first finding of this study was that the concurrent 12-week exercise could alleviate IR and counteract liver fat accumulation induced by an HFD. However, the evidence of exercise in the prevention of liver fat accumulation induced by an HFD still remains controversial. Some studies reported that exercise had no effect on liver lipid accumulation in HFD-induced obese rats [34, 35]. However, another study [36] demonstrated that concurrent exercise completely prevented hepatic steatosis induced by an HFD. The difference between these opposing findings might be owing to the fact that the latter study applied exercise at the same time as the induction of HFD, demonstrating that exercise should be conducted as early as possible to be effective.

On the other hand, the modalities of exercise treatment (i.e., exercise duration and exercise intensity) should also be considered to when evaluating its effects. Actually, there are no standard criteria for the modalities of an exercise regimen. It was reported that a concurrent 4-week exercise program failed to reverse hepatic steatosis induced by an HFD in rats [37]. This is probably because the exercise duration was too short to compensate for the metabolic disorder induced by the HFD. According to a recent study, a 12-week moderate aerobic exercise protocol proved to be effective in the management of T2DM and NAFLD progression in Otsuka Long-Evans Tokushima Fatty (OLETF) rats [38]. Our results were in accordance with the previous research [38], and showed that a moderate-intensity long-term exercise protocol was effective in suppressing fatty liver deposits and improving peripheral IR status. Notably, in the previously mentioned study, the exercise was started after the OLETF rats developed moderate NAFLD, so they could not completely reverse the disease condition. Thus, for it to have the greatest suppressive effect on IR and NAFLD, an exercise-regime should be applied as early as possible; also, the exercise should be at least a moderate intensity, and the regime should be adopted for the long term, as stated in the present study.

To gain insight into the concrete mechanism of how exercise prevents liver fat accumulation, we next measured the expressions of PPAR- $\gamma, \mathrm{CPT}-1$, and MCAD in the livers of the three groups. As shown in the present study, the expressions of PPAR- $\gamma$, CPT-l, and MCAD in the HFDE group were increased, and the values of TC, FFA, and IRI were decreased as compared with the HFD group, indicating that exercise exerted anti-lipid accumulation effects through PPAR- $\gamma$ / CPT-1 / MCAD signaling. These results are in accordance with the previous study's findings [39] that upregulation of PPAR evokes $\beta$-oxidation of fatty acids, increasing glucose uptake and catabolism, and in turn alleviates NALFD and improves IR. To our knowledge, most studies showed improved insulin sensitivity and lipid metabolism via PPAR- $\alpha$ target genes, CPT-1, and MCAD [40, 41], while few carried on with PPAR- $\gamma$ / CPT-1/ MCAD signaling

Table 2 Biochemical indicators of three groups after 12 weeks

\begin{tabular}{|c|c|c|c|c|c|c|c|c|}
\hline Groups & $\begin{array}{l}\text { TC } \\
(\mathrm{mmol} / \mathrm{l})\end{array}$ & $\begin{array}{l}\text { TG } \\
(\mathrm{mmol} / \mathrm{l})\end{array}$ & $\begin{array}{l}\mathrm{HDL} \\
(\mathrm{mmol} / \mathrm{l})\end{array}$ & $\begin{array}{l}\text { LDL } \\
(\mathrm{mmol} / \mathrm{l})\end{array}$ & $\begin{array}{l}\text { FFA } \\
(\mathrm{mmol} / \mathrm{l})\end{array}$ & $\begin{array}{l}\text { FIN } \\
(\mathrm{mlU} / \mathrm{ml})\end{array}$ & $\begin{array}{l}\text { FPG } \\
(\mathrm{mmol} / \mathrm{l})\end{array}$ & $|\mathrm{R}|$ \\
\hline $\mathrm{ND}(n=10)$ & $4.78 \pm 0.18$ & $0.57 \pm 0.11$ & $2.13 \pm 0.21$ & $0.94 \pm 0.23$ & $1.00 \pm 0.28$ & $4.43 \pm 1.30$ & $5.58 \pm 0.84$ & $1.11 \pm 0.17$ \\
\hline $\operatorname{HFD}(n=10)$ & $20.82 \pm 0.75^{*}$ & $1.12 \pm 0.49^{*}$ & $1.52 \pm 0.12$ & $8.31 \pm 0.44^{* *}$ & $1.47 \pm 0.27^{*}$ & $10.99 \pm 0.82^{* *}$ & $9.03 \pm 1.17^{* *}$ & $4.43 \pm 0.43^{* *}$ \\
\hline $\operatorname{HFDE}(n=10)$ & $12.95 \pm 0.53 \#$ & $0.67 \pm 0.19^{\#}$ & $2.11 \pm 0.38$ & $4.74 \pm 0.35^{\# \#}$ & $1.03 \pm 0.20^{\#}$ & $7.28 \pm 0.75^{\#}$ & $6.37 \pm 0.71^{\#}$ & $2.08 \pm 0.47^{\# \#}$ \\
\hline
\end{tabular}

TC total serum cholesterol, TG triglyceride, HDL high-density lipoprotein cholesterol, LDL low-density lipoprotein cholesterol, FFA free fatty acid, FIN fasting serum insulin level, $F P G$ fasting plasma glucose, IRI insulin resistance index. All the values are presented as Means \pm SD. ${ }^{*} p<0.05,{ }^{* *} p<0.01$, compared with ND group; ${ }^{\#}$ $p<0.05, \# p<0.01$, compared with HFD group 


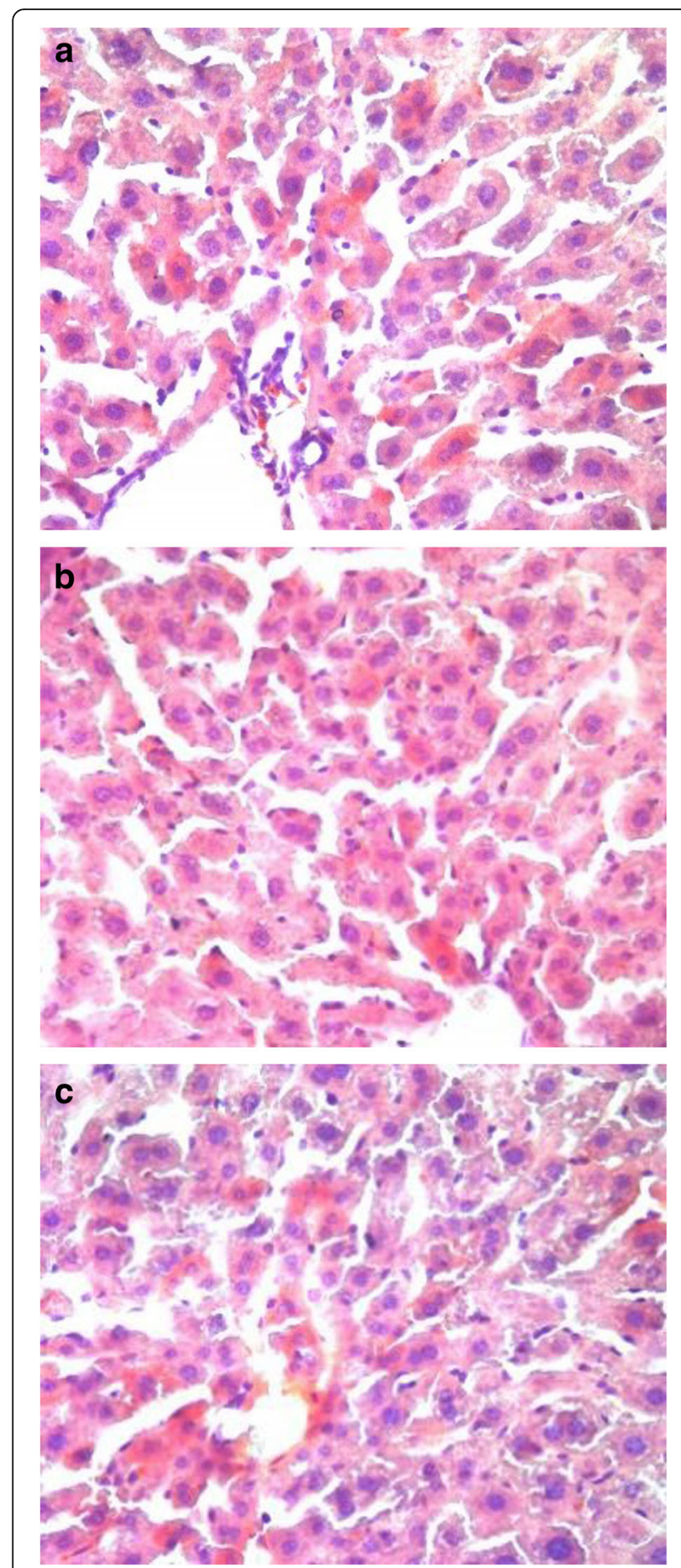

Fig. 2 Oil Red O staining of liver sections in ND, HFD, and HFDE groups (400x magnification). a Oil Red O staining of ND group. $\mathbf{b}$ Oil Red O staining of HFD group. $\mathbf{c}$ Oil Red O staining of HFDE group

in the liver. This was partially done because PPAR- $\gamma$ is mainly expressed in the adipose tissue, playing a vital role in the whole body lipid metabolism and insulin sensitivity [27]. However, under conditions of nonalcoholic fatty liver disease and nonalcoholic steatohepatitis, PPAR- $\gamma$ was obviously overexpressed in the liver and correlated with insulin sensitivity [42, 43]. It was argued that the full activation of PPAR- $\gamma$ triggered the transcription of lipogenic transcription factors, which favored obesity and NAFLD. However, the partial PPAR- $\gamma$ activation could lead to an increased adiponectin level and insulin sensitivity, thus it would be a benefit to NAFLD and IR [44, 45]. It was reported that telmisartan, partially activated PPAR- $\gamma$ combining with activating PPAR-alpha in the liver, could alleviate hepatic steatosis in mice that were fed an HFD [46]. In addition, telmisartan exerted beneficial effects on fatty livers in the treatment of human NAFLD when compared with losartan, highlighting the vital role of PPARs in the management of NAFLD [47]. Similarly, ragaglitazar, a dual PPAR-alpha/PPAR-gamma agonist, had beneficial effects on IR, hepatic steatosis and overweight induced by an HFD [48]. These drugs for NAFLD, including partially activated PPAR- $\gamma$, might mimic the effects of exercise on IR and hepatic steatosis. Moreover, evidence showed that increased PPAR- $\gamma$ could upregulate CPT-1 and improve de novo lipogenesis and liver steatosis in rodents fed an HFD [27]. Based on the findings of the present study, we speculated that the expression of PPAR- $\gamma$ was partially increased by long-term aerobic exercise, and it might concomitantly upregulate the expressions of CPT-1 and its target gene MCAD. This effect subsequently resulted in the alleviation of IR and NAFLD via enhanced fatty acid oxidation.

The highlight of this study is that the current study first provides insights on the effects of exercise on PPAR- $\gamma$ down- regulation of CPT-1 and MCAD in the livers of mice on an HFD. This study provides potential targets for the clinical treatment of NAFLD in conjunction with IR. However, there are some limitations to this study. First, the sample size used in the study was determined by the sample size used by previous researchers $[36,49]$.. Due to the limited samples in this study, there is an increased risk of lower statistical power, and some important effects of physical training may not be detected. Second, we only involved male mice in the present study. However, a large body of evidence showed that NAFLD was a sexual dimorphic disease; that is, the male sex was at a higher risk of NAFLD [50]. Thus, the physiopathological peculiarities of NAFLD in females should be taken into account, and a more robust number of observations should allow for addressing the role of exercise for the two genders. Last, we only observed the expressions of PPAR- $\gamma, \mathrm{CPT}-1$, and MCAD in the livers of mice with IR and NAFLD. Therefore, future research work should focus on the direct mechanism of exercise alleviation of IR and NAFLD in the liver tissue by regulating PPAR- $\gamma / \mathrm{CPT}-1 / \mathrm{MCAD}$ both in vivo 


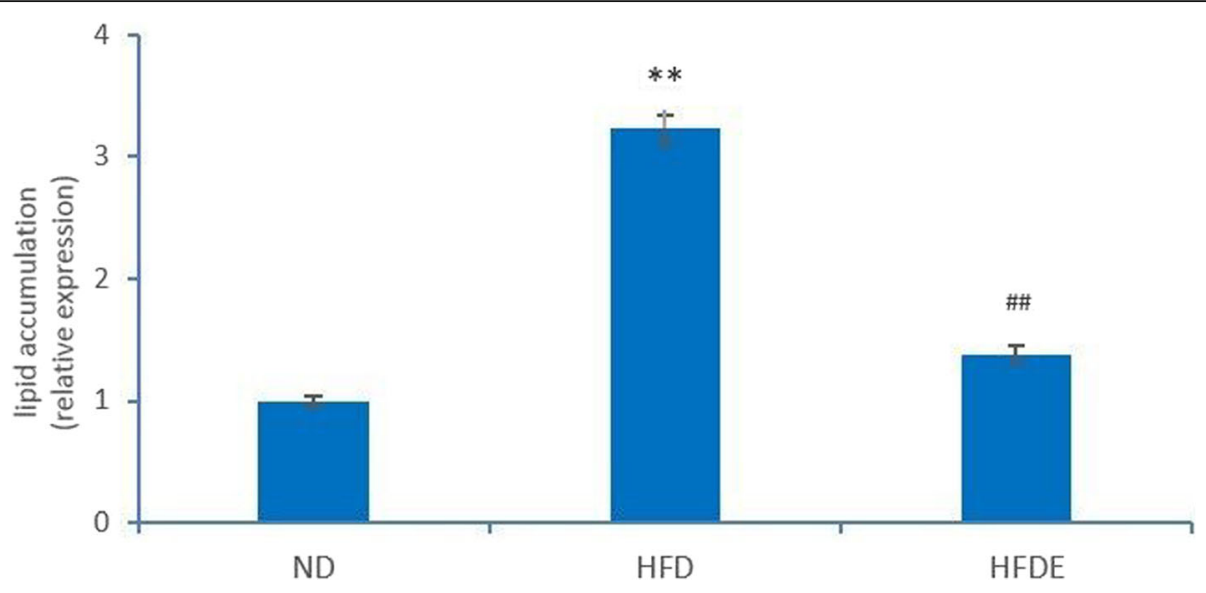

Fig. 3 Quantified intracellular fat drops were extracted using isopropanol and measured by a microplate reader at $510 \mathrm{~nm}$. The histogram shows the relative fold change compared with ND group (set as 1). Data are presented as Means \pm SD, $n=30$. ${ }^{* *} p<0.01$ vs ND group, ${ }^{\# \#} p<0.01$ vs HFD group

and in vitro. The use of PPAR- $\gamma$ knockout mice will clarify the exercise effects on IR and NAFLD via PPAR- $\gamma / C P T-1 /$ MCAD signaling.

\section{Conclusions}

Through a concurrent 12-week swimming training, lipid metabolism disorder and IR in the HFD ApoE-KO mice were significantly improved. The expressions of PPAR- $\gamma, \mathrm{CPT}$, and MCAD in the HFDE group were significantly increased, suggesting that swimming training can partially activate the PPAR- $\gamma$ expression in the liver tissue, concomitantly increase CPT-1 and MCAD levels, and finally alleviate the liver lipid disorders of IR and NAFLD.

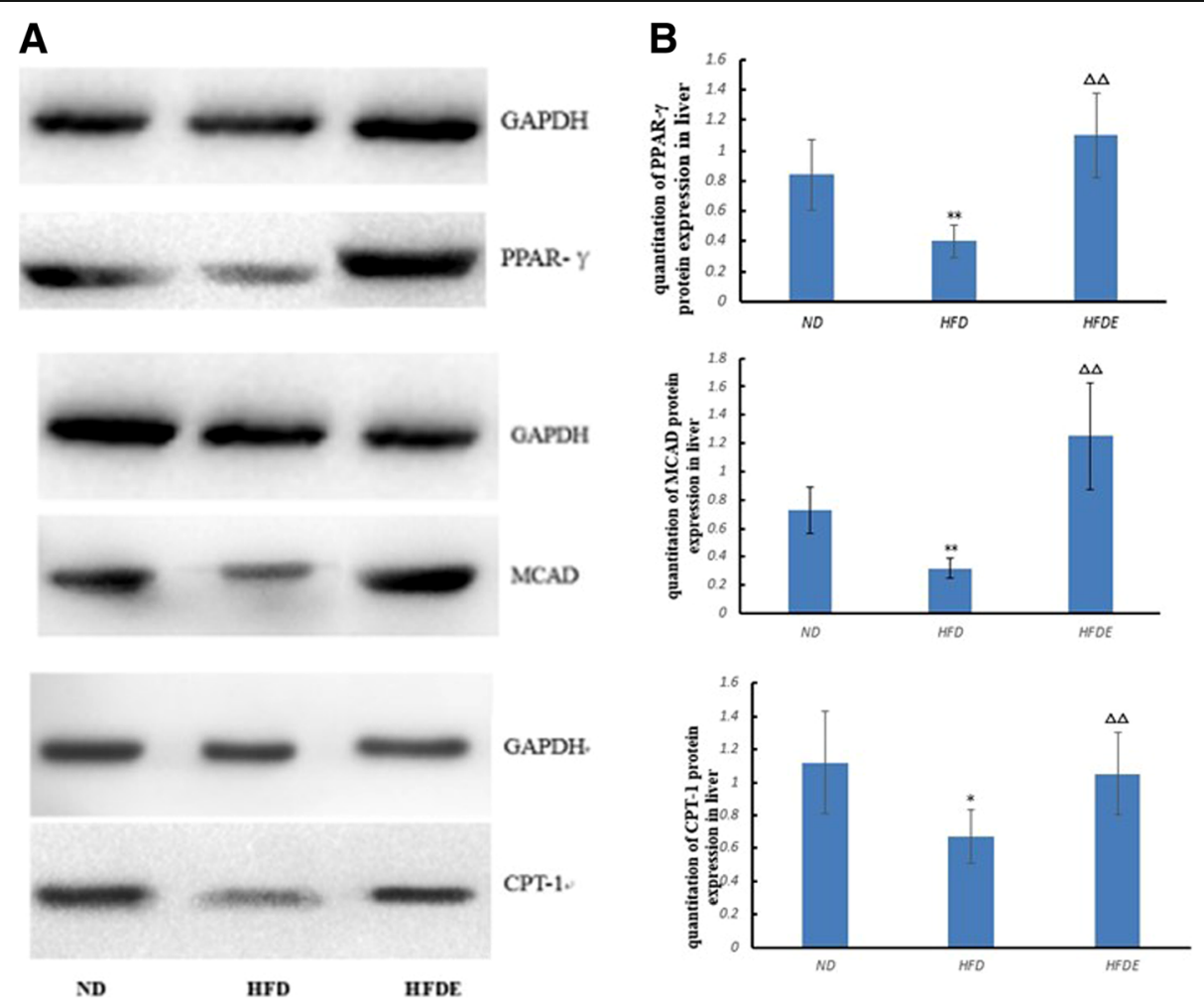

Fig. 4 Effects of exercise on the protein levels of PPAR-Y, MCAD and CPT-1 in the livers of ApoE-/- mice fed an HFD. a Western blot bands of PPAR-y, MCAD and CPT-1. b The relative protein expression levels were normalized by that of GAPDH (GAPDH, glyceraldehyde-3-phosphate dehydrogenase). ${ }^{*} P<0.05$ vs. ND; ${ }^{\Delta \Delta} P<0.01$ vs. HFD 


\section{Abbreviations}

ApoE-KO: Apolipoprotein E knock out; CPT-1: Carnitine palmitoyl transferase1; FFA: Free fatty acid; FFAs: Free fatty acids concentrations; FIN: Fasting serum insulin level; FPG: Fasting plasma glucose; GAPDH: Glyceraldehyde-3phosphate dehydrogenase; HDL: High-density lipoprotein cholesterol; HFD: High fat diet; HFDE: High fat diet plus exercise; IR: Insulin resistance; IRI: Insulin resistance index; LDL: Low-density lipoprotein cholesterol; MCAD: Medium-chain acyl-CoA dehydrogenase; NAFLD: Nonalcoholic fatty liver disease; NASH: Nonalcoholic steatohepatitis; ND: Normal diet; OLETF: Otsuka long-evans tokushima fatty; PPAR: Peroxisome proliferatoractivated receptors; SD: Standard deviation; T2DM: Type 2 diabetes mellitus; TC: Total serum cholesterol; TG: Triglyceride

\section{Acknowledgements}

Not applicable.

\section{Funding}

This research was by supported by the National Natural Science Foundation of China (Grant No. 81501955).

\section{Availability of data and materials}

All data generated or analyzed during this study are included in this published article.

\section{Authors' contributions}

YC conceived and designed the study. YC and FZ performed the experiments. YC and FZ analyzed and interpreted the data. FZ and YC contributed in writing the manuscript. All authors read and approved the final manuscript.

\section{Ethics approval and consent to participate}

The protocol of animal experiments was complied with the Guide for the Care and Use of Laboratory Animals formulated by the US National Institutes of Health (NIH Publication No. 85-23, Revised 1996) and approved by the Animal Care and Ethics Committee of Central South University, Changsha, China.

\section{Consent for publication}

Not applicable.

\section{Competing interests}

The authors declare that they have no competing interests.

\section{Publisher's Note}

Springer Nature remains neutral with regard to jurisdictional claims in published maps and institutional affiliations.

Received: 31 March 2018 Accepted: 27 November 2018

Published online: 05 January 2019

\section{References}

1. Choi D, Jung C, Mok J, et al. Effect of Dapagliflozin on alanine aminotransferase improvement in type 2 diabetes mellitus with nonalcoholic fatty liver disease. Endocrinol Metab. 2018;33(3):387-94.

2. Defronzo RA, Ferrannini E, Groop L, et al. Type 2 diabetes mellitus. Nat Rev Dis Primers. 2015;1(41):15019.

3. Yu J, Zheng J, Liu XF, et al. Exercise improved lipid metabolism and insulin sensitivity in rats fed a high-fat diet by regulating glucose transporter 4 (GLUT4) and musclin expression. Braz J Med Biol Res. 2016;49(5):e5129.

4. Wu CL, Zhao SP, Yu BL. Intracellular role of exchangeable apolipoproteins in energy homeostasis, obesity and non-alcoholic fatty liver disease. Biol Rev Camb Philos Soc. 2015;90(2):367-76

5. Targher G, Byrne CD, Lonardo A, et al. Non-alcoholic fatty liver disease and risk of incident cardiovascular disease: a meta-analysis. J Hepatol. 2016;65(3): 589-600.

6. Park SH, Kim BI, Yun JW, et al. Insulin resistance and C-reactive protein as independent risk factors for non-alcoholic fatty liver disease in non-obese Asian men. Eur J Gastroenterol Hepatol. 2004;19(6):694-8.

7. Lonardo A, Ballestri S, Marchesini G, et al. Nonalcoholic fatty liver disease: a precursor of the metabolic syndrome. Dig Liver Dis. 2015;47(3):181-90.

8. Boden G. Obesity, insulin resistance and free fatty acids. Curr Opin Endocrinol Diabetes Obes. 2011;18(2):139.
9. Li Z, Xue J, Chen P, Chen L, et al. Prevalence of nonalcoholic fatty liver disease in mainland of China: a meta-analysis of published studies. J Gastroenterol Hepatol. 2014;29(1):42-51.

10. Wei J, Lei GH, Fu L, et al. Association between dietary vitamin C intake and non-alcoholic fatty liver disease: a cross-sectional study among middle-aged and older adults. PLoS One. 2016;11(1):e147985.

11. Rcr M, Watt MJ. Hepatokines: linking nonalcoholic fatty liver disease and insulin resistance. Nat Rev Endocrinol. 2017;13(9):509-20.

12. Jean-Marc L, Abdolnaser P. NAFLD, estrogens, and physical exercise: the animal model. J Nutr Metab. 2012;3:914938.

13. Sun C, Huang F, Liu $X$, et al. miR-21 regulates triglyceride and cholesterol metabolism in non-alcoholic fatty liver disease by targeting HMGCR. Int J Mol Med. 2015:35(3):847-53.

14. Luo J, Xu L, Li J, et al. Nonalcoholic fatty liver disease as a potential risk factor of cardiovascular disease. Eur J Gastroenterol Hepatol. 2015:27(3):193.

15. Neuschwander Tetri BA. Nonalcoholic steatohepatitis and the metabolic syndrome. Am J Med Sci. 2005:330(6):326-35.

16. Lonardo A, Nascimbeni F, Targher G, et al. AISF position paper on nonalcoholic fatty liver disease (NAFLD): updates and future directions. Dig Liver Dis. 2017;49(5):471-83.

17. Lonardo A, Nascimbeni F, Maurantonio M, et al. Nonalcoholic fatty liver disease: evolving paradigms. World J Gastroenterol. 2017;23(36):6571-92.

18. Romero-Gómez M, Zelber-Sagi S, Trenell M. Treatment of NAFLD with diet, physical activity and exercise. J Hepatol. 2017;67(4):829-46.

19. Kvandová M, Majzúnová M, Dovinová I. The role of PPARgamma in cardiovascular diseases. Physiol Res. 2016;65(Supplementum 3):S343-63.

20. Tanaka N, Aoyama T, Kimura S, et al. Targeting nuclear receptors for the treatment of fatty liver disease. Pharmacol Ther. 2017;179:142-57.

21. Ballestri $S$, Nascimbeni F, Romagnoli D, et al. The role of nuclear receptors in the pathophysiology, natural course, and drug treatment of NAFLD in humans. Adv Ther. 2016;33(3):291-319.

22. Chen J. 15-Deoxy-delta 12, 14-prostaglandin J2 is a ligand for the adipocyte determination factor PPAR gamma. Cell. 1995;83(5):803.

23. Lehmann JM, Moore LB, Smith-Oliver TA, et al. An antidiabetic thiazolidinedione is a high affinity ligand for peroxisome proliferator-activated receptor gamma (PPAR gamma). J Biol Chem. 1995;270(22):12953-6.

24. Hammarstedt A, Andersson CX, Rotter SV, et al. The effect of PPARgamma ligands on the adipose tissue in insulin resistance. Prostaglandins Leukot Essent Fatty Acids. 2005;73(73):65-75

25. Pellieux C, Aasum E, Larsen TS, et al. Overexpression of angiotensinogen in the myocardium induces downregulation of the fatty acid oxidation pathway. J Mol Cell Cardiol. 2006;41(3):459-66.

26. Neuschwandertetri BA, Brunt EM, Wehmeier KR, et al. Improved nonalcoholic steatohepatitis after 48 weeks of treatment with the PPARgamma ligand rosiglitazone. Hepatology. 2003;38(4):1008-17.

27. Mello T, Materozzi M, Galli A. PPARs and mitochondrial metabolism: from NAFLD to HCC. PPAR Res. 2016;2016:1-18.

28. Zheng $\mathrm{H}$, Li S, Liang $M$, et al. A novel agonist of PPAR-y based on barbituric acid alleviates the development of non-alcoholic fatty liver disease by regulating adipocytokine expression and preventing insulin resistance. Eur J Pharmacol. 2011;659(2-3):244-51.

29. Fliegner D, Westermann D, Riad A, et al. Up-regulation of PPARgamma in myocardial infarction. Eur J Heart Fail. 2008;10(1):30.

30. Gilde AJ, van der Lee KAJM, Willemsen PHM, et al. Peroxisome proliferatoractivated receptor (PPAR) $a$ and PPAR $/ \delta$, but not PPARY, modulate the expression of genes involved in cardiac lipid metabolism. Circ Res. 2003;92(5):518.

31. Gulick T, Cresci S, Caira T, et al. The peroxisome proliferator-activated receptor regulates mitochondrial fatty acid oxidative enzyme gene expression. Proc Natl Acad Sci. 1994;91(23):11012.

32. Tous M, Ferré N, Camps J, et al. Feeding apolipoprotein E-knockout mice with cholesterol and fat enriched diets may be a model of non-alcoholic steatohepatitis. Mol Cell Biochem. 2005;268(1):53-8.

33. Schierwagen R, Maybüchen L, Zimmer S, et al. Seven weeks of Western diet in apolipoprotein-E-deficient mice induce metabolic syndrome and nonalcoholic steatohepatitis with liver fibrosis. Sci Rep. 2015;5(1):12931.

34. Estadella D, Oyama LM, Dâmaso AR, et al. Effect of palatable hyperlipidic diet on lipid metabolism of sedentary and exercised rats. Nutrition. 2004; 20(2):218-24.

35. Burneiko RC, Diniz YS, Galhardi CM, et al. Interaction of hypercaloric diet and physical exercise on lipid profile, oxidative stress and antioxidant defenses. Food Chem Toxicol. 2006;44(7):1167-72. 
36. Gauthier M, Couturier K, Latour J, et al. Concurrent exercise prevents high-fat-dietinduced macrovesicular hepatic steatosis. J Appl Physiol. 2003;94(6):2127-34.

37. Straczkowski M, Kowalska I, Dzienis-Straczkowska S, et al. The effect of exercise training on glucose tolerance and skeletal muscle triacylglycerol content in rats fed with a high-fat diet. Diabetes Metab. 2001;27(1):19-23.

38. Linden MA, Fletcher JA, Morris EM, et al. Combining metformin and aerobic exercise training in the treatment of type 2 diabetes and NAFLD in OLETF rats. Am J Physiol Endocrinol Metab. 2014;306(3):E300.

39. Domínguez-Avila JA, González-Aguilar GA, Alvarez-Parrilla E, et al. Modulation of PPAR expression and activity in response to polyphenolic compounds in high fat diets. Int J Mol Sci. 2016;17(7):1002.

40. Chen X, Zhang F, Gong Q, et al. Hepatic ATF6 increases fatty acid oxidation to attenuate hepatic steatosis in mice through peroxisome proliferatoractivated receptor alpha. Diabetes. 2016;65(7):b151637.

41. Han H, Qiu F, Zhao H, et al. Dietary flaxseed oil prevents Western-type dietinduced nonalcoholic fatty liver disease in apolipoprotein-E knockout mice. Oxid Med Cell Longev. 2017;2017(9):1-13.

42. Inoue $M$, Ohtake T, Motomura W, et al. Increased expression of PPARy in high fat diet-induced liver steatosis in mice. Biochem Biophys Res Commun. 2005;336(1):215-22.

43. Zhang YL, Hernandez-Ono A, Siri P, et al. Aberrant hepatic expression of PPARgamma2 stimulates hepatic lipogenesis in a mouse model of obesity, insulin resistance, dyslipidemia, and hepatic steatosis. J Biol Chem. 2006; 281(49):37603-15.

44. Zhang Y, Gu M, Cai W, et al. Dietary component isorhamnetin is a PPARY antagonist and ameliorates metabolic disorders induced by diet or leptin deficiency. Sci Rep. 2016;6(1):19288.

45. Souza-Mello V. Peroxisome proliferator-activated receptors as targets to treat non-alcoholic fatty liver disease. World J Hepatol. 2015;7(8):1012.

46. Souzamello V, Gregório BM, Cardosodelemos FS, et al. Comparative effects of telmisartan, sitagliptin and metformin alone or in combination on obesity, insulin resistance, and liver and pancreas remodelling in C57BL/6 mice fed on a very high-fat diet. Clin Sci. 2010;119(6):239-50.

47. Hirata T, Tomita K, Kawai T, et al. Effect of Telmisartan or Losartan for Treatment of Nonalcoholic Fatty Liver Disease: Fatty Liver Protection Trial by Telmisartan or Losartan Study (FANTASY). Int J Endocrinol. 2013;2013(1):587140.

48. Ye JM, Iglesias $M A$, Watson $D G$, et al. PPARalpha /gamma ragaglitazar eliminates fatty liver and enhances insulin action in fat-fed rats in the absence of hepatomegaly. Am J Physiol Endocrinol Metab. 2003;284(3):E531.

49. Marques CMM, Motta VF, Torres TS, et al. Beneficial effects of exercise training (treadmill) on insulin resistance and nonalcoholic fatty liver disease in high-fat fed C57BL/6 mice. Braz J Med Biol Res. 2010;43(5):467-75.

50. Ballestri S, Nascimbeni F, Baldelli E, et al. NAFLD as a sexual dimorphic disease: role of gender and reproductive status in the development and progression of nonalcoholic fatty liver disease and inherent cardiovascular risk. Adv Ther. 2017:34(6):1291-326.

Ready to submit your research? Choose BMC and benefit from:

- fast, convenient online submission

- thorough peer review by experienced researchers in your field

- rapid publication on acceptance

- support for research data, including large and complex data types

- gold Open Access which fosters wider collaboration and increased citations

- maximum visibility for your research: over $100 \mathrm{M}$ website views per year

At $\mathrm{BMC}$, research is always in progress.

Learn more biomedcentral.com/submissions 